examine ICT prescribing in other UK regions to identify if this is a more widespread problem.

\section{S12 UNSCHEDULED HEALTHCARE RESOURCE UTILISATION AND HEALTH-RELATED QUALITY OF LIFE BEFORE AND AFTER OMALIZUMAB INITIATION IN UK CLINICAL PRACTICE: THE APEX STUDY}

doi:10.1136/thoraxjnl-2011-201054b.12

\begin{abstract}
${ }^{1} \mathrm{~N}$ Barnes, ${ }^{2} \mathrm{~A}$ Radwan, ${ }^{3} \mathrm{~F}$ Percival. ${ }^{1}$ Barts and The London NHS Trust, London, UK; ${ }^{2}$ Novartis Pharmaceuticals UK Limited, Frimley, UK; ${ }^{3} \mathrm{pH}$ Associates Limited, Marlow, $U K$
\end{abstract}

Objectives The efficacy and safety of omalizumab for the treatment of severe persistent allergic asthma have been demonstrated in randomised controlled clinical trials. However, there are limited "real world" data on its effects on healthcare resource utilisation or health-related quality of life (QoL) in UK clinical practice.

Methods A 10 centre retrospective observational study (APEX) compared 12 months pre-vs 12 months post-omalizumab initiation in patients aged $=12$ years with severe persistent allergic asthma. All patients received $=1$ dose of omalizumab. Patients who had received omalizumab in a clinical trial were excluded. Hospital records were reviewed to obtain data on hospital resource use and routinely used QoL measures for example, Asthma Quality of Life Questionnaire (AOLO) at baseline (pre-omalizumab), 16 weeks and up to 12 months following omalizumab initiation.

Results Mean in-patient hospital admissions fell by $61 \%$ from 1.30 to $0.51(\mathrm{p}<0.001)$ and mean in-patient bed days fell by $70 \%$ from 9.10 to $2.74(p<0.001)$ per patient. In the subgroup of patients hospitalised for asthma in the 12 months pre-omalizumab $(n=81)$, mean in-patient hospital admissions fell by $70 \%$ from 2.19 to 0.65 $(p<0.001)$ and mean in-patient bed days fell by $74 \%$ from 14.86 to $3.83(p<0.001)$ per patient. Similarly, mean Accident and Emergency department attendances fell by $70 \%$ from 1.52 per patient in the 12 months pre-omalizumab to 0.46 in the 12 months post-omalizumab $(p<0.001)$. Other resource use, such as outpatient attendances (excluding visits made solely for omalizumab administration), nurse appointments and telephone consultations remained unchanged following omalizumab initiation. OoL data were not available for all patients at every time point. However, where data were available, mean AOLO scores increased from 3.09 at baseline to 5.01 at 16 weeks $(n=90)$ and to 5.22 at 12 months $(n=29)$.

Conclusions Treatment with omalizumab is associated with a clinically and statistically significant reduction in unplanned hospital resource utilisation and improvements in patients' QoL.

\section{Unravelling airway infection in COPD S13 DIFFERENTIATED HUMAN RHINOVIRUS LOADS IN STABLE
COPD AND AT EXACERBATION}

doi:10.1136/thoraxjnl-2011-201054b.13

S N George, A J Mackay, A R C Patel, R J Sapsford, D S Garcha, G C Donaldson, J A Wedzicha. University College London, London, UK

Introduction Human rhinoviruses (HRV) are the main aetiological agents of virus-associated COPD exacerbations (Seemungal et al, 2001). However the importance of the level of viral load as a trigger for naturally occurring exacerbations is not fully understood. We aimed to assess and quantify HRV prevalence and load in stable and exacerbating patients from the London COPD cohort.

Methods A real-time qPCR protocol was utilised to detect HRV presence and quantify load in sputum samples taken at baseline $(n=58)$ and at COPD exacerbation onset $(n=57)$. COPD patients were defined as stable if exacerbation-free for at least 42 days since the previous exacerbation and more than 14 days before the next. Exacerbations were defined using our usual symptom criteria; an increase in respiratory symptoms for two consecutive days, with at least one symptom being a major symptom; dyspnoea, sputum purulence or volume and the other a major or minor symptom; wheeze, cold sore throat, and cough (Anthonisen criteria). A $\chi^{2}$ test was used to compare HRV prevalence of the two disease phases, and an independent-samples $t$ test was used to compare the differences in viral load.

Results Sixty-four patients provided 115 sputum samples: mean age 70.5 years ( $\mathrm{SD} \pm 8.1) ; \mathrm{FEV}_{1} 45.8 \%$ predicted $( \pm 20.0 \%)$; current smoker $31.3 \%$. There is a significantly higher prevalence of HRV at exacerbation onset, $31.6 \%(18 / 57)$ compared to baseline $15.5 \%$ (9/ 58) $(\mathrm{p}=0.042)$. Similarly, the mean viral load was significantly greater at exacerbation onset $1.70( \pm 1.67) \log _{10} \mathrm{pfu} / \mathrm{ml}$ than baseline $0.30( \pm 0.69) \quad \log _{10} \mathrm{pfu} / \mathrm{ml}(\mathrm{p}=0.025)$, exhibiting a 25 -fold increase in viral load from baseline to exacerbation. $6.9 \%(4 / 58)$ of patients were positive for HRV at baseline only, 26.3\% (15/57) at exacerbation only and $8.8 \%(4 / 45)$ at both time points $(p=0.006)$.

Conclusions HRV load is significantly greater at COPD exacerbation than when detected in the stable state. This emphasises the importance of HRV as a key trigger of COPD exacerbations. HRV can be detected in the stable state; however the loads are very low suggesting asymptomatic carriage rather than chronic infection.

\section{S14 HISTONE DEACETYLASE ACTIVITY IS REDUCED IN COPD SUBJECTS DURING RHINOVIRUS INDUCED EXACERBATIONS}

doi:10.1136/thoraxjnl-2011-201054b.14

J I M Footitt, P Mallia, M B Trujillo-Torralbo, A Durham, I M Adcock, S L Johnston. NHLI, Imperial College, London, UK

Introduction and Objectives Histone deacetylase (HDAC) enzymes have a role in suppressing inflammatory gene transcription. There is evidence for reduced HDAC activity in COPD which correlates with the severity of airflow obstruction. Increased inflammation found during exacerbations of COPD may result from a reduction in HDAC activity but this has not been studied in virus induced exacerbations. We sought to investigate HDAC activity following rhinovirus infection in an experimental model of COPD exacerbations.

Methods Experimental rhinovirus challenge was performed in GOLD stage II COPD subjects and non-obstructed control smokers and non-smokers. Rhinovirus infection was confirmed with quantitative PCR performed on nasal lavage and sputum samples collected at baseline and days 3, 5, 9, 12, 15, 21 and 42 post virus inoculation. Sputum macrophages were isolated by adhesion and HDAC2 isoenzyme immunoprecipitated, prior to performing a HDAC activity assay.

Results 11 non-smokers (NS), 10 smokers (Smk) and 9 COPD subjects were recruited. The mean (SEM) \% predicted baseline $\mathrm{FEV}_{1}$ was 103 (4), 98 (4) and 69 (2)\% for NS, Smk and COPD respectively. At baseline there was no difference in HDAC2 activity between the three study groups, the geometric mean $(95 \% \mathrm{CI})$ activity was NS 22.39 (12.45 to 40.25); Smk 22.91 (18.28 to 28.71) and COPD 48.98 (26.04 to 92.13 ) ( $p=0.095)$. Following rhinovirus infection, in NS HDAC2 activity increased, in Smk it remained largely unchanged and there was a fall from baseline levels in COPD subjects at all time points, Abstract S14 figure 1.

Conclusions HDAC2 activity was not reduced in stable COPD subjects compared to controls. This may reflect the mild disease state of the study population. During a rhinovirus induced 